\title{
Efeitos de um programa de atividade física sobre os sintomas depressivos e a qualidade de vida de idosos com demência de Alzheimer
}

CDD. 20.ed. 362.196831

613.7

\author{
Heloisa Schievano GROPPO* \\ Carla Manuela Crispim NASCIMENTO* \\ Florindo STELLA* \\ Sebastião GOBBI* \\ Mérlyn Mércia OLIANI*
}

*Universidade Estadual

Paulista - Rio Claro.

\begin{abstract}
Resumo
0 objetivo do estudo foi analisar os efeitos de um programa de exercícios físicos sobre os sintomas depressivos e a percepção da Qualidade de vida (OV) de pacientes com Doença de Alzheimer (DA) e de seus cuidadores. Seis idosas realizaram um programa de exercícios físicos durante seis meses, outras seis compuseram o grupo controle. 0 Mini-Exame do Estado Mental, a Escala de Qualidade de Vida e a Escala de Depressão em Geriatria foram aplicados para avaliação das variáveis. Os dados foram analisados através de uma ANOVA "two-way" e correlação de Pearson, com nivel de significância de $5 \%(p \leq 0,05)$. Os resultados mostraram que o programa proposto pode auxiliar na redução dos sintomas depressivos de pacientes com DA, mas não promoveu melhoras significativas na percepção da QV destes pacientes e nem de seus cuidadores. Entretanto menores comprometimentos da percepção da qualidade de vida foram observados em pacientes e cuidadores que eram fisicamente ativos.
\end{abstract}

Unitermos: Demência de Alzheimer; Sintomas depressivos; Qualidade de vida; Atividade física.

\section{Introdução}

A doença de Alzheimer (DA) é a forma mais comum de demência caracterizada por múltiplos déficits cognitivos decorrentes de um processo neurodegenerativo progressivo e irreversível que comumente compromete a funcionalidade, com perda de autonomia na realização das atividades cotidianas e reflexos negativos na Qualidade de Vida (QV) (Souza, Galante \& Figueiredo, 2003; Tatsumi, Nakaaki, ToriI, Shinagawa, Watanabe, Murata, Sato, Mimura \& FuruKawa, 2009).

Os processos patológicos decorrentes da progressão da DA podem gerar profundas inabilidades e fragilidade afetando dimensōes afetivas, psíquicas e funcionais que intensificam o comprometimento da $\mathrm{QV}$, pois comprometem a adaptação psicossocial e a interação do idoso com o ambiente (ABREU, ForLENZA \& BARROS, 2005). O nível de dependência do paciente é uma medida global que reflete o nível de severidade e a QV (ANDERSEN,
Wittrup-Jensen, LolK, Andersen \& KraGh-Sorensen, 2004). Além do impacto negativo sobre a QV do paciente, a demência pode prejudicar de maneira intensa a QV dos cuidadores destes pacientes. Essa redução da QV do cuidador ocorre devido a sentimentos como angústia ou aflição que aparecem ao mesmo tempo em que os sintomas depressivos surgem no paciente.

Um crescente número de evidências científicas relacionadas ao tratamento da DA atualmente vem sendo relacionado não somente às formas de intervenção farmacológica, mas com grande enfoque nas formas complementares de abordagens não-farmacológicas. Estas abordagens associadas ao medicamento têm como objetivo proporcionar uma possível atenuação do comprometimento cognitivo e retardar o avanço dos comprometimentos cognitivos e neuropsiquiátricos e o exercício físico vem se mostrando uma alternativa eficaz e de baixo custo 
com bons resultados nas esferas cognitivas e afetivas destes pacientes (Kramer, Erickson \& Colcombe, 2006; Teri, LogsDOn \& McCurry, 2008).

Christofoletti, Oliani, Bucken-Gobbi, GobBi, Beinotti e Stella (2011) realizaram um estudo de revisão que indicava que intervenções nãofarmacológicas associadas a pratica de atividades físicas consistem em importantes ferramentas para a promoção de benefícios psicossociais, status afetivo,

\section{Método}

\section{Amostra}

Foram recrutadas para fazerem parte do estudo 16 idosas com diagnóstico clínico de DA segundo os critérios clínicos do Manual Diagnóstico e Estatístico de Transtornos Mentais (DSM-IV-R) (APA, 2002) nas fases leve e moderada da doença de acordo com o Escore Clínico de Demência (Hughes, Berg, Danziger, Coben \& Martin, 1982) Entretanto, deste total, três participantes do grupo de treinamento não concluíram o Protocolo de Treinamento com a frequência mínima exigida para serem inseridas na amostra, e uma idosa do grupo controle não participou da avaliação final devido ao agravamento da doença. A amostra final do estudo, portanto, contava com 12 participantes com idade média de 79,25 \pm 7,23 anos e escolaridade média de 8,33 $\pm 2,99$ anos. Seis idosas utilizavam apenas a rivastigmina no tratamento da DA, três delas, apenas memantina, e outras três faziam uso de ambas as medicações.

Todas as pacientes residiam na cidade de Rio Claro, SP, Brasil e foram distribuídas em dois grupos: 1) um grupo treinamento (GT; $n=6)$, submetido a um programa de exercícios físicos sistematizado por um período de seis meses. Foram considerados para análise estatística apenas os pacientes que obtiveram uma frequência mínima de 75\%; de participação e; 2) um grupo controle (GC; $n=6)$ que no referido período do estudo não realizou nenhum tipo de prática atividade motora sistematizada. Foram excluídos do estudo pacientes em estágio grave da DA, devido à impossibilidade de cumprirem, tanto o protocolo de avaliação quanto o programa de exercícios físicos proposto. Também foram excluídos da amostra quaisquer participantes que apresentassem outras comorbidades neuropsiquiátricas. estresse do cuidador e saúde física. Entretanto poucos estudos dedicaram-se a análises longitudinais para verificarem os efeitos crônicos da prática regular de exercícios físicos em pacientes com DA.

Diante disto, o objetivo do presente estudo foi verificar os efeitos de um programa de exercícios físicos sobre os sintomas depressivos de idosos com DA e ainda, sobre a percepção de qualidade de vida destes pacientes e de seus cuidadores.

\section{Protocolo do programa de exercícios físicos}

O GT realizou um programa de exercício físico generalizado e sistematizado. Foram realizadas três sessōes semanais em dias não consecutivos por um período de seis meses (24 semanas). Dentro do protocolo foram incluídos exercícios que enfatizavam o trabalho dos componentes da capacidade funcional (agilidade, equilíbrio, resistência de força e capacidade aeróbia) associados às tarefas cognitivas (por exemplo: contagem regressiva, reconhecimentos de formas, cores, tarefas de fluência verbal, etc.). Para os componentes funcionais, a agilidade foi desenvolvida com exercícios envolvendo mudança de direção e mobilidade; o equilíbrio foi trabalhado por meio de circuitos com estimulações nos sistemas somatossensorial, vestibular e visual com materiais de diferentes densidades e dimensões de superfícies para realizar as tarefas motoras.; para resistência de força o protocolo envolvia prioritariamente exercícios com os principais e grandes grupos musculares com séries longas (15 a 20 repetiçōes) e baixa sobrecarga. Materiais foram inseridos à medida que o treinamento avançava a fim de realizar a sobrecarga. Inicialmente as atividades utilizavam o peso do próprio corpo e passaram a ter incrementos mensalmente por meio de equipamentos como bastões, bolas, halteres, caneleiras e "thera-band", bem como havia sobrecarga de volume para evitar intensidades muito altas que expusessem o idoso a algum risco. Atividades de marcha que estimulassem a mobilidade e deambulação dos participantes eram realizadas de maneira leve para o trabalho cardiorrespiratório. Os aumentos das intensidades eram realizados assim que se observava que a atividade proposta não era capaz de manter a frequência cardíaca máxima (FCM) dos participantes no limiar a ser estimulado $(60-80 \%$ FCM). Foi realizado o controle da frequência cardíaca 
dos participantes por meio do uso de frequencímetros durante todo o período de treinamento e as atividades deveriam ser mantidas no limiar proposto de FCM de acordo com a capacidade do paciente para manter-se ativo com segurança.

Concomitantemente ao treinamento físico, materiais com cores e formas diferentes, músicas e jogos que associassem a atividade motora a outros estímulos (verbais, visuais, táteis e auditivos) eram inseridos ao longo da sessão. O GT final foi composto pelos idosos que permaneceram pelas 24 semanas no programa, tendo um mínimo de $75 \%$ de presença nas aulas. Tal porcentagem de faltas foi permitida devido a eventuais problemas de saúde ou distúrbios de comportamento que pudessem comprometer a participação eventualmente do paciente no programa de exercícios físicos.

O GC não participou de nenhum programa de programa de exercícios físicos ou atividades sistematizadas similares (fisioterapia, terapia ocupacional, etc.). Ambos os grupos mantinham o tratamento farmacológico por todo o período da investigação.

Os cuidadores dos pacientes participantes do estudo assinaram um Termo de Consentimento Livre e Esclarecido, segundo as normas estabelecidas pela resolução 196/96 do Conselho Nacional de Saúde às pesquisas envolvendo seres humanos. O presente estudo foi aprovado pelo Comitê de ética em pesquisa do Instituto de Biociências, UNESP, Campus de Rio Claro (CEP-IB-UNESP), protocolo 2469.

\section{Instrumentos para coleta de dados}

Os dados foram coletados por meio de questionários respondidos pelos pacientes e, quando se fazia necessário para atender ao protocolo de aplicação, pelos respectivos cuidadores. Tais informaçôes foram coletadas no início e após o período de seis meses.

As funçôes cognitivas foram avaliadas por meio do Mini-Exame do Estado Mental (MEEM) (FolsteIN, Folstein \& McHugh, 1975). O MEEM é composto por questôes agrupadas em sete categorias, cada uma delas planejada com o objetivo de avaliar funções cognitivas específicas. São elas: orientação para tempo, orientação para local, registro de três palavras, atenção e cálculo, recordação das três palavras, linguagem e capacidade construtiva visual. O escore do MEEM varia de 0 a 30 pontos, sendo que valores mais baixos apontam para possível déficit cognitivo. O diagnóstico para declínio cognitivo é determinado a partir da pontuação sugerida por BRUCKI, Nitrini, CARAMELLI, BertolucCi e OKAMOto (2003). Os autores padronizaram o uso deste instrumento no Brasil segundo o grau de escolaridade. Para analfabetos considera-se o corte de 19 pontos; com um a três anos de escolaridade, 23 pontos; quatro a sete anos, 24 pontos e acima de sete anos de instrução, 28 pontos. Pacientes que apresentarem valores iguais ou acima do ponto de corte são considerados normais cognitivamente. Aqueles que apresentarem valores abaixo do ponto de corte apresentam provável déficit cognitivo.

A Escala de Depressão em Geriatria (EDG) (Yesavage, Brink, Rose, Lum, Huang, Adey \& LEIRER, 1982) é uma escala simples, amplamente utilizada e validada como instrumento de diagnóstico de depressão em pacientes idosos. É um teste elaborado a partir de 30 itens que mais fortemente se correlacionam com diagnostico de depressão. A pontuação final corresponde a um escore formado pela somatória de perguntas específicas e escores acima de 10 pontos indicam a possibilidade de quadros depressivos. Esses itens, em conjunto, mostraram boa acurácia diagnóstica, com sensibilidade, especificidade e confiabilidade adequadas. AlmeIDA e Almeida (1999a, 1999b) demonstraram que a versão brasileira da GDS oferece uma medida válida para o diagnóstico de episódio depressivo maior de acordo com os critérios da Classificação Internacional de Doenças (CID-10) e DSM-IV. A avaliação da consistência interna da escala através do coeficiente alfa de Cronbach revelou índices de confiabilidade de 0,81 para a GDS. Esses resultados indicam que a forma curta da GDS oferece uma medida válida para a detecção de casos de depressão entre idosos.

A escala de qualidade de vida (EQV) (LOGSDON, Gibbons, McCurry \& Terry, 2002) é baseada em um questionário, compostos por três sub-escalas que avaliam 13 questôes fechadas sobre a análise dos diversos elementos que compõem a qualidade de vida, classificando-os em ruim, regular, bom ou excelente. $\mathrm{O}$ primeiro questionário avalia a percepção do paciente sobre sua QV (QVpac); o segundo, a versão do cuidador (familiar) sobre a QV do paciente (QVvcuid); e, o terceiro, a percepção de $\mathrm{QV}$ do cuidador sobre si mesmo (QVcuid). Para obter o escore do paciente, é preciso realizar um cálculo matemático (multiplicar a primeira sub-escala por dois, somar este resultado à segunda sub-escala e dividir o resultado global por três). Quanto maior o número de pontos obtidos, melhor éa percepção de QV. Noveli, Nitriñ e CARAmElLi (2010) validaram a $E Q V$ na língua portuguesa e concluíram que o instrumento tem excelente estabilidade e confiabilidade (alfa $=0,81 / 0,85 / 0,84$ para: paciente, familiar cuidador e visão do cuidador sobre a QV do paciente, respectivamente). Os índices de correlação 
encontrados na avaliação intra-examinador foram 0,87/0,95/0,95 ( $\mathrm{P}<0,001)$. Na avaliação interexaminador, os índices foram 0,76/0,96/0,93 ( $\mathrm{P}<$ $0,001)$.

\section{Análise dos dados}

O teste de Shapiro-Wilk demonstrou que os dados apresentavam uma distribuição normal, portanto os

\section{Resultados}

Os valores obtidos na avaliaçãao por meio da escala de qualidade de vida e dos sintomas depressivos encontramse na TABELA 1. A análise de variância para medidas repetidas mostrou que os pacientes do GT tiveram uma redução nos sintomas depressivos, enquanto que o GC apresentou piora para os escores do mesmo teste.

Em relação à escala de $\mathrm{QV}$, os pacientes do $\mathrm{GC}$ tiveram um declínio mais acentuado na pontuação que os pacientes do GT. Para as sub-escalas respondidas pelos cuidadores, foi possível observar um discreto comprometimento na pontuação do GC e a manutenção dos escores preliminares no GT.

A análise de correlação de Pearson foi realizada para verificar o grau de associação entre a percepção de $\mathrm{QV}$ dos pacientes com a intensidade dos sintomas depressivos dos mesmos. A relação entre as variáveis foi verificada primeiramente na amostra total. Em um segundo momento, foi feita uma análise dos grupos separadamente, a fim de verificar se há alguma diferença no grau de associação destas variáveis moduladas pela prática de atividade física.

$\mathrm{Na}$ análise da amostra total, os coeficientes de correlação de Pearson obtidos foram:

$-\mathrm{r}=-0,68^{*}(\mathrm{p}=0,015)$, entre QVcuid_0 e EDG_0;

$-\mathrm{r}=-0,523(\mathrm{p}=0,081)$, entre QVpac_0 e EDG_0;

$-r=-0,89^{*}(p=0,000)$, entre QVpac_6 e EDG_6. resultados foram expressos por meio de médias e desviospadrão. A Análise de Variância para medidas repetidas (ANOVA "two-way") foi utilizada para verificar a interação entre momentos (pré e pós-treinamento) e grupos (controle e treinamento) para os sintomas depressivos e a qualidade de vida de pacientes e cuidadores. O coeficiente de correlação de Pearson foi utilizado para verificar a relação entre as variáveis avaliadas. Admitiu-se para todos os testes um nível de significância de 5\% ( $\mathrm{p} \leq 0,05)$.

Para uma análise dos grupos separadamente, $\mathrm{o}$ coeficiente de correlação de Pearson apontou:

Para o GC, os valores de $r$ foram:

$-r=-0,83^{*}(p=0,041)$ entre EDG_0 eQVCUID_0

$-r=-0,88^{*}(p=0,021)$ entre EDG_6e QVPAC_0

$-r=-0,84^{*}(p=0,034)$ entre EDG_6e QVPAC_6

Para QV, ainda pode-se observar uma correlação entre os valores de pré e pós e entre sub-escalas para o GC:

- $r=0,93^{*}(p=0,007)$ entre QVVCUI_0 e QVVCUI_6

- $r=0,89^{*}(p=0,016)$ entre QVVCUI_0 e QVCUI_0

$-r=0,92^{*}(p=0,009)$ entre QVCUID_0 e QVCUID_6

Para o GT, foi observado apenas $\mathrm{r}=0,861^{*}(\mathrm{p}=$ 0,28) entre QVPAC_0 e QVVCUID_0.

A ANOVA "two-way" mostrou haver interação significativa entre grupos (GC e GT) e os momentos (pré e pós-treinamento) para os dados relativos a EDG. Para $\mathrm{QV}$, não foram encontradas interações significativas.
*A ANOVA "two-way" mostrou uma interação significativa entre grupos e momentos para os valores da EDG $(\mathrm{F}=9,42 ; p=0,01)$. Não houve interação significativa para QVpac e QV cuid.

TABELA 1 - Médias edesvios-padrão das variáveis Mini-ExamedoEstadoMental(MEEM), Escala deDepressãoem Geriatria (EDG), QualidadedeVida do paciente(QV_pac), Qualidadedevida docuidador(QV_vcuid)eQualidadedevida do paciente pela visão do cuidador (QV_cuid), para os grupos controle (GC) e treinameento (GI).

\begin{tabular}{ccccc}
\hline & & GC & & \multicolumn{2}{c}{ GI } \\
\hline & Pré & Pós & Pré & Pós \\
\hline MEEM & 14 & 11,66 & 17,8 & 16,16 \\
EGD & 6,33 & 9 & 8,33 & $4 *$ \\
QV_pac & 39,5 & 33 & 39,8 & 37,6 \\
QV_vcuid & 34,3 & 32,1 & 37,5 & 37,1 \\
QV_cuid & 34,3 & 32,16 & 37,5 & 37,16 \\
\hline
\end{tabular}




\section{Discussão}

O presente estudo pretendeu investigar os efeitos de um programa, regular e sistematizado, de exercícios físicos generalizados, sobre sintomas depressivos e percepção da QV de pacientes com DA e ainda, o impacto que este programa apresenta na percepção da QV de cuidadores de idosos com DA.

Com relação aos sintomas depressivos, foi possível notar uma melhora nos pacientes que passaram por treinamento e uma piora nos pacientes que não participaram de nenhum tipo de programa regular e sistematizado de estimulação motora. Essa informação corrobora com alguns estudos encontrados na literatura.

Blumenthal, Babyak, Moore, Craighead, Herman, Khatri, Waugh, Napolitano, Forman, AppelBAUM, DORAISWAMU e KRISHNAN (1999) observaram que a atividade física aeróbia pode ser considerada como uma alternativa não-farmacológica no tratamento de sintomas depressivos em idosos. Ao final do estudo, o grupo que realizou três sessōes semanais de exercícios físicos obteve reduçoes significativas nos sintomas depressivos, resultados similares com volumes de treinamento também similares aos do conduzido nesta investigação. Antunes, Stella, Santos, Bueno e MelLo (2005) encontraram que seis meses de exercício aeróbio são capazes de reduzir sintomas de ansiedade e depressão em idosos e similarmente, um estudo realizado por Deslandes, Moraes, Alves, Pompeu, Silveira, Mouta, Arcoverde, Ribeiro, Cagy, Piedade, Laks e CoutinHo (2010), mostrou que o treinamento aeróbio realizado duas vezes por semana provoca redução nos sintomas depressivos de idosos com depressão. Nosso estudo foi conduzido com uma intervenção que tinha por base o estímulo prioritariamente do metabolismo aeróbio, mas não trabalhava exclusivamente a atividade de 'caminhada'. O programa de exercícios generalizados proposto visava também realizar estímulos em diversas esferas da funcionalidade e, além disto, foi capaz de promover melhoras significativas nos sintomas afetivos de idosos com DA, similarmente a outras intervençôes menos específicas.

Diversos mecanismos plausíveis para explicar como o exercício afeta a depressão têm sido propostos. Atualmente, a prática de atividade física regular é vista como um benefício, pois o paciente depressivo, envolvido com esta prática, pode ter como resultado o "feedback" positivo de outras pessoas aumentando a sua auto-estima. $\mathrm{O}$ ato de exercitar-se pode servir como uma distração de pensamentos negativos e o domínio de novos hábitos podem ser importantes. $\mathrm{O}$ contato social propiciado pelo contexto da prática da atividade física pode ser um importante mecanismo, bem como a atividade física causa efeitos fisiológicos como mudanças na concentração de endorfinas e monoaminas, que podem agir beneficamente sobre a depressão, diminuindo a ansiedade, tensão e estresse (LAWLOR \& HOPKER 2001). Estudos conduzidos com a mesma base de treinamento utilizado nesta investigação verificaram benefícios significativos na redução de distúrbios neuropsiquiátricos (NASCImento, Teixeira, Gobbi, Gobbi \& Stella, 2012) de indivíduos com DA após a realização de um protocolo de treinamento similar ao deste estudo com reflexos positivos na redução da sobrecarga do cuidador (Christofoletti et al., 2011). Nossos achados corroboram com tais investigações acerca dos benefícios da atividade física para os pacientes e ainda com extensão para seus cuidadores. Ainda no contexto afetivo, Vital, Hernandez, Stein, Garuffi, Corazza, Andrade, Costa e Stella (2012) verificaram que pacientes com DA fisicamente ativos apresentaram menores intensidades de sintomas depressivos em relação aos seus pares sedentários. Tendo em vista que dentre os distúrbios neuropsiquiátricos observados em pacientes com DA, a depressão é o mais comum com uma prevalência estimada, no Brasil, de 38.3\% (Tatsch, Bottino, Azevedo, Hototian, Moscoso, Folquitto, Scalco \& Louza, 2006). Deste forma, levando-se em conta os reflexos que a intensificação destes distúrbios com a progressão da doença podem representar na perda da funcionalidade do paciente com aumento do estresse e sobrecarga do cuidador, nosso programa de exercícios apresenta efeitos positivos tanto na redução dos transtornos depressivos com impacto sobre uma melhora significativa na percepção da QV tanto do paciente quanto do seu cuidador, conforme foi discutido em um estudo de revisão por Mello, Boscolo, Esteves e Tufik (2005).

A partir da análise dos dados, é possível verificar que o nível de sintomas depressivos interfere de forma negativa na percepção da $\mathrm{QV}$ de pacientes com $\mathrm{DA}$, podendo refletir na QV do seu cuidador. XAVIER, Ferraz, Bertolucci, Poyares e Moriguchi (2003), indicaram também um grau de associação entre pior percepção de QV (mais insatisfeitos) e maiores sintomas depressivos em indivíduos idosos. A análise de grupos pela correlação de Pearson reforça tais achados confirmando que os sintomas depressivos podem apresentar influência significativa na percepção de qualidade de vida também em idosos com DA. Isso sugere que a redução significativa apresentada nos 
sintomas depressivos após o período de prática de um programa de exercícios físicos sistematizado pode ter reduzido a relação dose-resposta entre os sintomas depressivos e redução da QV, ou seja, a diminuição de uma das variáveis dependentes gerou uma resposta significativa no grau de associação apresentado por estes indivíduos, indicando a importância do controle deste tipo de alteração neuropsiquiátrica na percepção da QV de pacientes e cuidadores.

Logsdon, Gibbons, McCurry e Teri (2004) encontraram que síntomas depressivos do paciente são associados com pior QV do paciente, de acordo com a visão do cuidador. A QV é uma variável multidimensional sendo influenciada por inúmeras condições e interações de fatores ambientais, fisicos e psico-sociais. A atividade física tem se mostrado uma alternativa eficaz na promoção de melhoras em muitas destas esferas que interagem na percepção da QV também em pessoas idosas com DA (HeYN, 2003).

Os valores de correlação encontrados para QV nos mostram que a visão do cuidador sobre a $\mathrm{QV}$ apresenta um bom grau de associação com a visão que o paciente percebe de sua QV. Isso nos sugere que apesar dos prejuízos cognitivos decorrentes da DA, o paciente é capaz de ter a percepção do quanto sua QV pode estar sendo afetada e ainda, nos permite observar que o processo de dependência funcional e agravamento do quadro clínico da DA interferem de forma significativa na QV de seus cuidadores. SHIN, Carter, Masterman, Fairbank e Cummings (2005) também investigaram o impacto dos distúrbios neuropsiquiátricos na QV de pacientes e cuidadores e verificaram que intervenções que possam beneficiar os pacientes com a redução destes sintomas podem melhorar a percepção de QV de pacientes e cuidadores. Portanto, o programa proposto mostra-se como uma alternativa de tratamento coadjuvante não-farmacológica viável na atenuação dos sintomas depressivos com impactos positivos na QV.

Tendo em conta que a QV é uma noção subjetiva que leva em conta muitas dimensōes, nosso estudo demonstra que o comprometimento decorrente da DA e o comprometimento nas dimensōes afetivas tem um papel significativo na percepção da QV. Tais achados também podem ser confirmados quando levamos em conta a correlação encontrada entre presença e intensidade de sintomas depressivos nos pacientes com a QV do cuidador, indicando o papel de interação que esta dimensão neuropsíquica. Em revisão de literatura, Antunes, SANTOS, CASSILHAS, Santos, Bueno e Mello (2006) concluíram que pessoas moderadamente ativas têm menor risco de ser acometidas por desordens mentais do que as sedentárias, e que indivíduos fisicamente ativos provavelmente possuem um processamento cognitivo mais rápido. Também encontraram significativo aumento do desempenho físico de pessoas idosas com déficit cognitivo e demência com a prática de exercício físico. Esta melhora no desempenho físico pode representar uma redução na dependência para realização das atividades da vida diária, que normalmente requer uma maior supervisão por parte dos cuidadores e frequentemente leva estas pessoas a um aumento na sobrecarga, estresse e impactos em suas saúdes física e mental. Estes resultados corroboram com o estudo de Rolland, Pillard, Klapouszczak, Reynish, Thomas, Andrieu, Riviere e Velas (2007) que avaliaram os efeitos de um programa de exercícios de flexibilidade, equilibrio e força e encontraram melhoras significativas na redução dos sintomas neuropsiquiatricos e no desempenho das atividades da vida diária.

Christofoletti et al. (2011) encontraram que cuidadores cujos pacientes com DA praticavam mais atividade física, sofriam menor desgaste mental (avaliado pelo Inventário Neuropsiquiátrico) que cuidadores de pacientes com maiores níveis de atividade física. A não significância estatística pode ter ocorrido pelo grande número de fatores que influenciam a QV (multidimensionalidade). Cada indivíduo tem sua própria percepção do que é boa $Q V$, e esta pode ser diretamente influenciada por alguns fatores como classe social e estado emocional (Novelli, Dal RoVERE, Nitrini \& Caramelli, 2005).

Portanto, a atividade física pode não ter sido suficiente para promover uma melhora da QV. Além disso, a doença tem um grande avanço em diversas dimensões cognitivas em um período de seis meses, que pôde não ter sido identificado pelos escores do MEEM. Baixos escores no MEEM podem representar maiores necessidades de cuidados e perda mais acentuada de autonomia do paciente, podendo refletir com maior impacto em seus cuidadores (Novelli, Melandri, Bertolotti \& Vidotto, 2009).

Diante dos danos irreversíveis da DA ao paciente, e das dificuldades no tratamento farmacológico, farse-ão necessários maiores estudos com terapias não farmacológicas alternativas que objetivem atenuar o avanço do quadro do processo degenerativo e de suas consequências.

Conclui-se que programa de exercícios físicos generalizados realizado de maneira regular e sistematizada por um período de seis meses é uma alternativa possível, de baixo custo e eficaz na redução de sintomas depressivos de idosos com DA. Apesar de 
não terem sido demonstradas melhoras significativas na QV de idosos com DA e de seus cuidadores, o impacto decorrente do avanço da doença sobre esta variável parece ocorrer de maneira menos acentuada nos pacientes fisicamente ativos em comparação com os do grupo controle. Mais estudos se fazem necessários para uma melhor investigação dos efeitos da atividade física nessas variáveis.

\begin{abstract}
Effects of a physical activity program on depressive symptoms and quality of life of elderly with Alzheimer's dementia

The aim of the study was to analyze the effects of a physical exercises' program on depressive symptoms and quality of life (OL) in elderly with Alzheimer's Disease (AD) and OL of their caregivers. Six elderly women performed a physical exercises' program with six months of duration, and the other six composed the control group. The Mini-Mental State Examination, the Quality of Life Scale and the Geriatric Depression Scale were applied to evaluate the variables. Data were analyzed by Analyze of Variance (Two-way) and Pearson's correlations. The level of significance was set at $5 \%(p \leq 0.05)$. The results showed that the physical exercises' program proposed could aid on the reduction of depressive symptoms of AD patients. However less worsening was observed on $\mathrm{OL}$ of patients and caregivers from the physically active group.
\end{abstract}

UnItERMs: Alzheimer's dementia; Depressive symptoms; Quality of life; Physical activity.

\title{
Resumen
}

Efectos de un programa de actividad física en los síntomas depressivos y la calidad de vida del ancianos con demencia de Alzheimer

El objetivo del estudio fue analizar los efectos de un programa de ejercicios en los síntomas depresivos y la percepción de calidad de vida (CV) de los pacientes con Demencia de Alzheimer (DA) y sus cuidadores. Seis personas ancianas realizaran un programa de ejercicio físico por un periodo del seis meses, otro grupo estaba formado por el grupo de control. El Mini Examen del Estado Mental, la Escala de Calidad de Vida y la Escala de Depresión Geriátrica fueran utilizados para evaluar las variables. Los datos fueron analizados por análisis de varianza de dos entradas (ANOVA de dos vías) y la correlación de Pearson, con un nivel de significancia de $5 \%(p \leq 0,05)$. Los resultados muestran que el programa propuesto puede ayudar en la reducción de los sintomas depresivos en pacientes con DA, pero no promove mejoras significativas en la percepción de la calidad de vida de estos pacientes ni sus cuidadores. Sin embargo menos daños de la percepción de calidad de vida se observaron en los pacientes y los cuidadores que eran físicamente activos.

Palabras clave: Demencia de Alzheimer; Síntomas depresivos; Calidad de vida; Actividad física.

\section{Referências}

ABREU, I.D.; FORLENZA, O.V.; BARROS, H.L. Demência de Alzheimer: correlação entre memória e autonomia. Revista de Psiquiatria Clínica, São Paulo, v.32, n.1, p.131-36, 2005.

ALMEIDA, O.P.; ALMEIDA, S.A. Reliability of the Brazilian version of the geriatric depression scale (GDS) short form. Arquivos de Neuro-psiquiatria, São Paulo, v.57, n.2B, p.421-26, 1999a. 
Short versions of the geriatric depression scale: a study of their validity for the diagnosis of a major depressive episode according to ICD-10 and DSM-IV. International Journal of Geriatric Psychiatry, Chichester, v.14, n.10, p.858-65, 1999b. AMERICAN PSYCHIATRIC ASSOCIATION (APA). Manual diagnóstico e estatístico de transtornos mentais: DSM-IV-TR. Porto Alegre: Artmed, 2002. p.168-77.

ANDERSEN, C.K.; WITTRUP-JENSEN, K.U.; LOLK, A.; ANDERSEN, K.; KRAGH-SORENSEN, P. Ability to perform activities of daily living is the main factor affecting quality of life in patients with dementia.Health Quality Life Outcomes, London, v.2, p.52, 2004.

ANTUNES, H.K.; STELLA, S.G.; SANTOS, R.F; BUENO, O.F.; DE MELLO, M.T. Depression, anxiety and quality of life scores in seniors after an endurance exercise program. Revista Brasileira de Psiquiatria, São Paulo, v.27, n.4, p.266-71, 2005. ANTUNES, H.K.M.; SANTOS, R.F.; CASSILHAS, F.; SANTOS, R.V.T.; BUENO, O.F.A.; MELLO, M.T. Exercício físico e função cognitiva: uma revisão. Revista Brasileira de Medicina do Esporte, São Paulo, v.12, n.2, p.108-14, 2006. BLUMENTHAL, J.A.; BABYAK, M.A.; MOORE, K.A.; CRAIGHEAD, W.E.; HERMAN, S.; KHATRI, P.; WAUGH, R.; NAPOLITANO, M.A.; FORMAN, L.M.; APPELBAUM, M.; DORAISWAMY, P.M.; KRISHNAN, K.R. Effects of exercise training on older patients with major depression. Archives of Internal Medicine, Chicago, v.159, n.19, p.2349356, 1999.

BRUCKI, S.M.; NITRINI, R.; CARAMELLI, P.; BERTOLUCCI, P.H.; OKAMOTO, I.H. Suggestions for utilization of the mini-mental state examination in Brazil. Arquivos de Neuro-psiquiatria, São Paulo, v.61, n.3B, p.777-81, 2003. CHRISTOFOLETTI, G.; OLIANI, M.M.; BUCKEN-GOBBI, L.T.; GOBBI, S.; BEINOTTI, F.; STELLA, F. Physical activity attenuates neuropsychiatric disturbances and caregiver burden in patients with dementia.Clinics, São Paulo, v.66, n.4, p.613-18, 2011.

DESLANDES, A.C.; MORAES, H.; ALVES, H.; POMPEU, F. A.; SILVEIRA, H.; MOUTA, R.; ARCOVERDE, C.; RIBEIRO, P.; CAGY, M.; PIEDADE, R.A.; LAKS, J.; COUTINHO, E.S. Effect of aerobic training on EEG alpha asymmetry and depressive symptoms in the elderly: a 1-year follow-up study. Brazilian Journal of Medical and Biological Research, Ribeirão Preto, v.43, n.6, p.585-92, 2010.

FOLSTEIN, M.F.; FOLSTEIN, S.E.; McHUGH, P.R. Mini-mental state: a practical method for grading the cognitive state of patients for the clinician. Journal of Psychiatric Research, Oxford, v.12, n.3, p.189-98, 1975.

HEYN, P. The effect of a multisensory exercise program on engagement, behavior, and selected physiological indexes in persons with dementia. American Journal of Alzheimers Disease and Other Dementias, Weston, v.18, n.4, p.247-51, 2003. HUGHES, C.P.; BERG, L.; DANZIGER, W.L.; COBEN, L.A.; MARTIN, R.L. A new clinical scale for the staging of dementia. Brazilian Journal Psychiatry, Rio de Janeiro, v.140, p.566-72, 1982.

KRAMER, A.F.; ERICKSON, K.I.; COLCOMBE, S.J. Exercise, cognition, and the aging brain. Journal of Applied Physiology, Washington, v.101, n.4, p.1237-42, 2006.

LAWLOR, D.A.; HOPKER, S.W. The effectiveness of exercise as an intervention in the management of depression: systematic review and meta-regression analysis of randomised controlled trials. BMJ, London, v.322, n.7289, p.763-67, 2001. LOGSDON, R.G.; GIBBONS, L.E.; McCURRY, S.M.; TERI, L. Assessing changes in quality of life in Alzheimer's disease. Neurobiology of Aging, Fayetteville, v.25, 2004. Supplement 2.

Assessing quality of life in older adults with cognitive impairment. Psychosomatic Medicine, New York, v.64, n.3, p. $510-19,2002$.

MELLO, M.T.; BOSCOLO, R.A.; ESTEVES, A.M.; TUFIK, S. O exercício físico e os aspectos psicobiológicos. Revista Brasileira de Medicina no Esporte, São Paulo, v.11, n.3, p.203-07, 2005.

NASCIMENTO, C.M.; TEIXEIRA, C.V.; GOBBI, L.T.; GOBBI, S.; STELLA, F. A controlled clinical trial on the effects of exercise on neuropsychiatric disorders and instrumental activities in women with Alzheimer's disease. Revista Brasileira de Fisioterapia, São Paulo, v.16, n.3, p.197-204, 2012.

NOVELLI, B.; MELANDRI, D.; BERTOLOTTI, G.; VIDOTTO, G. Quality of life impact as outcome in burns patients. Giornale Italiano di Medicina del Lavoro ed Ergonomia, Pavia, v.31, p.A58-63, 2009. Supplement A.

NOVELLI, M.M.; DAL ROVERE, H.H.; NITRINI, R.; CARAMELLI, P. Cross-cultural adaptation of the quality of life assessment scale on Alzheimer disease. Arquivos de Neuropsiquiatria, São Paulo, v.63, n.2A, p.201-06, 2005.

NOVELLI, M.M.; NITRINI, R.; CARAMELLI, P. Validation of the Brazilian version of the quality of life scale for patients with Alzheimer's disease and their caregivers (QOL-AD). Aging and Mental Health, Abindgon, v.14, n.5, p.624-31, 2010. ROLLAND, Y.; PILLARD, F.; KLAPOUSZCZAK, A.; REYNISH, E.; THOMAS, D.; ANDRIEU, S.; RIVIERE, D.; VELLAS, B. Exercise program for nursing home residents with Alzheimer's disease: a 1-year randomized, controlled trial. Journal of the American Geriatrics Society, Baltimore, v.55, n.2, p.158-65, 2007. 
SHIN, I.S.; CARTER, M.; MASTERMAN, D.; FAIRBANKS, L.; CUMMINGS, J.L. Neuropsychiatric symptoms and quality of life in Alzheimer disease. American Journal of Geriatric Psychiatry, Washington, v.13, n.6, p.469-74, 2005. SOUZA, L.; GALANTE, H.; FIGUEIREDO, D. Qualidade de vida e bem-estar dos idosos: um estudo exploratório na população portuguesa. Revista Saúde Pública, São Paulo, v.37, n.3, p.364-71, 2003.

TATSCH, M.F.; BOTTINO, C.M.; AZEVEDO, D.; HOTOTIAN, S.R.; MOSCOSO, M.A.; FOLQUITTO, J.C.; SCALCO, A.Z.; LOUZA, M.R. Neuropsychiatric symptoms in Alzheimer disease and cognitively impaired, nondemented elderly from a community-based sample in Brazil: prevalence and relationship with dementia severity. American Journal of Geriatric Psychiatry, Washington, v.14, n.5, p.438-45, 2006.

TATSUMI, H.; NAKAAKI, S.; TORII, K.; SHINAGAWA, Y.; WATANABE, N.; MURATA, Y.; SATO, J.; MIMURA, M.; FURUKAWA, T.A. Neuropsychiatric symptoms predict change in quality of life of Alzheimer disease patients: a twoyear follow-up study. Psychiatry and Clinical Neurosciences, Carlton, v.63, n.3, p.374-84, 2009.

TERI, L.; LOGSDON, R.G.; McCURRY, S.M. Exercise interventions for dementia and cognitive impairment: the Seattle Protocols. The Journal of Nutrition, Health \& Aging, New York, v.12, n.6, p.391-94, 2008.

VITAL, T.M.; HERNANDEZ, S.S.; STEIN, A.M.; GARUFFI, M.; CORAZZA, D.I.; ANDRADE, L.P.; COSTA, J.L.; STELLA, F. Depressive symptoms and level of physical activity in patients with Alzheimer's disease. Geriatrics and Gerontology International, Tokyo, v.12, n.4, p.637-42, 2012.

XAVIER, F.M.F.; FERRAZ, M.P.T.; BERTOLUCCI, P.; POYARES, D.; MORIGUCHI, E.H. Episódio depressivo maior, prevalência e impacto sobre qualidade de vida, sono e cognição em octogenários. Revista Brasileira de Psiquiatria, São Paulo, v.23, n.2, p.62-70, 2003.

YESAVAGE, J.A.; BRINK, T.L.; ROSE, T.L.; LUM, O.; HUANG, V.; ADEY, M.; LEIRER, V.O. Development and validation of a geriatric depression screening scale: a preliminary report. Journal of Psychiatric Research, Oxford, v.17, n.1, p.37-49, 1982.

\begin{tabular}{r|r} 
ENDEREÇO & \\
Heloisa Schievano Groppo & Recebido para publicação: 24/03/2009 \\
Av. Doutor Morato, 967 & Revisão: 31/o5/2012 \\
Universidade Estadual Paulista - Campus Rio Claro & Aceito: 14/06/2012 \\
13405-260 - Piracicaba - SP - BRASIL & \\
e-mail: hgroppo@gmail.com & \\
&
\end{tabular}

